\title{
artigo
}

\section{Evidências do Tratamento de Eletroacupuntura em Mulheres com Incontinência Urinária de Esforço: Uma revisão integrativa}

\author{
Evidences of Electroacupuncture Treatment in Women with Stress Urinary Incontinence: an integrative review \\ Evidencia del Tratamiento con Electroacupuntura en Mujeres con Incontinencia Urinaria de Esfuerzo: una revisión integrativa
}

\begin{abstract}
RESUMO
Objetivo: Analisar as evidências do tratamento com eletroacupuntura em mulheres com incontinência urinária de esforço. Métodos: Revisão bibliográfica integrativa utilizando artigos científicos indexados nas bases de dados MEDLINE, LILACS, SciELO e PUBMED através dos descritores: "eletroacupuncture" e "stress urinary incontinence" constantes nos Descritores em Ciências da Saúde e o operador booleano AND. Os artigos obtidos foram os publicados entre 2009 e 2019. 0 estudo foi executado de maio a junho de 2019. Resultados: Foram encontrados 20 artigos no total nas bases de dados, restando 5 artigos para leitura na íntegra, sendo incluídos somente 3 que tinham relação direta com o tema. Constatou-se que o tratamento da incontinência urinária com a eletroacupuntura parece ser válido, principalmente nos casos mais leves, com menores quantidades de perdas da urina. Entretanto, devido poucos estudos, ainda não está bem definido um protocolo dos pontos de acupuntura assim como dos parâmetros da eletroacupuntura.
\end{abstract}

DESCRITORES: Eletroacupuntura; Incontinência Urinária por Estresse.

\section{ABSTRACT}

Objective: To analyze the evidence of treatment with electroacupuncture in women with stress urinary incontinence. Methods: Integrative bibliographic review using scientific articles indexed in the MEDLINE, LILACS, SciELO and PUBMED databases through the descriptors: "eletroacupuncture" and "stress urinary incontinence" in the Health Sciences Descriptors and the Boolean operator AND. The articles obtained were those published between 2009 and 2019. The study was carried out from May to June 2019. Results: A complete of 20 articles were found in the databases, leaving 5 articles for full reading, including only 3 that were related direct with the theme. It was found that the treatment of urinary incontinence with electroacupuncture seems to be valid, especially in cases with less urine loss. However, with few studies, an acupuncture point protocol as well as electroacupuncture parameters is not well defined.

DESCRIPTORS: Eletroacupuncture; Stress Urinary Incontinence.

\section{RESUMEN}

Objetivo: Analizar la evidencia del tratamiento con electroacupuntura en mujeres con incontinencia urinaria de esfuerzo. Métodos: Revisión bibliográfica integrativa utilizando artículos científicos indexados en las bases de datos MEDLINE, LILACS, SciELO y PUBMED a través de los descriptores: "electroacupuntura" e "incontinencia urinaria de esfuerzo" en los Descriptores de Ciencias de la Salud y el operador booleano AND. Los artículos obtenidos fueron los publicados entre 2009 y 2019. El estudio se realizó de mayo a junio de 2019. Resultados: Se encontraron un total de 20 artículos en las bases de datos, quedando 5 artículos para lectura completa, incluyendo solo 3 que estaban relacionados directo con el tema. Se encontró que el tratamiento de la incontinencia urinaria con electroacupuntura parece ser válido, especialmente en los casos con menor pérdida de orina. Sin embargo, con pocos estudios, un protocolo de puntos de acupuntura y los parámetros de electroacupuntura no están bien definidos.

DESCRIPTORES: Electroacupuntura; Incontinencia Urinaria de Esfuerzo.

RECEBIDO EM: 01/10/2020 APROVADO EM: 10/12/2020

\section{Patricia Cosentino Carvalho}

Fisioterapeuta Pélvica no HESFA/UFRJ, Mestre em Educação Física (UFRJ) Pós graduada em Acupuntura (UNIBAHIA) e em Biomecânica (UFRJ).

ORCID: 0000-0002-7840-4502 


\section{Raphael Neves Barreiros}

Enfermeiro. Mestre em Enfermagem pela Universidade Federal do Estado do Rio de Janeiro (UNIRIO); Pós Graduado em Acupuntura (UNIBAHIA).

ORCID: 0000-0002-0272-3187

\section{Luana Borges Dutra}

Enfermeira. Mestre em Enfermagem pela Universidade Federal do Estado do Rio de Janeiro (UNIRIO); Pós Graduada em Acupuntura (UNIBAHIA).

ORCID: 0000-0002-6132-3255

\section{Ricardo José Oliveira Mouta}

Doutor em Enfermagem. Professor Adjunto da Faculdade do Departamento Materno Infantil da Faculdade de Enfermagem da Universidade do Estado do Rio de Janeiro (UERJ).

ORCID: 0000-0002-1284-971X

\section{Thiago Quinellato Louro}

Enfermeiro, Pós Doutor em Enfermagem, Doutor em Ciências - PPGENFBIO/UNIRIO. Professor Adjunto II do Departamento de Enfermagem do Instituto de Humanidades em Saúde da Universidade Federal Fluminense (UFF). Coordenador do Grupo de Pesquisa: Laboratório de Pesquisa e Experimentação para o Ensino e Cuidado em Enfermagem - L@PETECEnf / UFF.

ORCID: 0000-0001-8371-628X

\section{INTRODUÇÃO}

A incontinência urinária (IU) é a perda involuntária de urina que causa um impacto direto na qualidade de vida das pessoas. Sua prevalência apresenta grande variabilidade, mas geralmente apontando as mulheres como a população mais acometida. Entre os principais fatores que podem influenciar para essa perda estão a gestação, idade, obesidade e as cirurgias ginecológicas $^{1}$. Para se definir o tipo de incontinência urinária, muito depende da avaliação clínica da paciente, dos sintomas e queixas apresentados e em alguns casos do exame de urodinâmica ${ }^{2}$.

A incontinência urinária de esforço (IUE) éum dos tipos de IU, sendo determinada como a queixa por qualquer perda involuntária de urina que ocorre devido à um esforço físico, como na tosse ou espirro, por um aumento da pressão intra-abdominal e incompetência dos músculos do diafragma pélvico².

A acupuntura é uma técnica milenar baseada na Medicina Tradicional Chinesa (MTC) que usa agulhas em pontos da superfície do corpo que possibilitam regular funções orgânicas. Nessa visão, a IUE seria principalmente o resultado da deficiência de Qi dos Rins que poderia afetar disfunção da Bexiga em controlar a micção ${ }^{3}$. Maciocia ${ }^{4}$ definiu o Qi como a energia que se apresenta tanto no nível físico como no espiritual. Algo que possa ser material e imaterial ao mesmo tempo e se manifestar nas formas do mais rarefeito até denso.

A eletroacupuntura é uma técnica dentro da prática da acupuntura que utiliza estímulos elétricos para movimentar as agulhas, substituindo os estímulos manuais do terapeuta e permitindo descrever os eletroestímulos que serão empregados nas agulhas 5 . O objetivo desse estudo foi elaborar uma revisão integrativa para compreender quais as evidências científicas atuais utilizando a eletroacupuntura no tratamento de mulheres com incontinência urinária de esforço (IUE).

\section{MÉTODO}

Trata-se de um estudo de revisão integrativa da literatura que é um método de pesquisa que permite a busca, a avaliação crítica e a síntese das evidências do tema investigado, sendo o seu produto o estado atual do conhecimento do tema investigado ${ }^{6}$ permitindo assim a capacidade de sistematização de conhecimentos científicos para o pesquisador ter a aproximação da temática que deseja estudar ${ }^{7}$. Para a elaboração deste estudo foram seguidas seis etapas distintas, sendo elas: 1) Identificação do tema e seleção da hipótese ou questão norteadora; 2) determinação de critérios para inclusão e exclusão de estudos; 3) categorização dos estudos; 4) avaliação dos estudos incluídos na revisão; 5) interpretação dos resultados e 6) síntese/apresentação do conhecimento ${ }^{6}$.
Para a identificação da questão da pesquisa, foi utilizada a estratégia PICO8. Trata-se de uma ferramenta que mantém o foco na pesquisa e é utilizada para a formulação da pergunta, na qual "P" (população ou proble$\mathrm{ma}$ ) referindo-se às mulheres com incontinência urinária de esforço; "I" (intervenção) a eletroacupuntura; "C" (comparação) não se aplica e "O" (desfecho esperado) melhoria dos sintomas da incontinência.

Portanto, para responder a questão da revisão:" Quais são as evidências do tratamento da incontinência urinária de esforço usando a eletroacupuntura abordados nos artigos publicados em periódicos nos últimos 10 anos?" O levantamento das publicações científicas foi realizado por meio dos descritores em base de dados e bibliotecas virtuais. O período da busca ocorreu de maio à junho de 2019. Foram consultadas as seguintes bases de dados eletrônicos: Biblioteca virtual em saúde (BVS) nas bases de dados da $\mathrm{Li}$ teratura Latino Americana e do Caribe em Ciências da Saúde (LILACS) e da Literatura Internacional em Ciências da Saúde (MEDLINE); Scientific Eletronic Library on Line (SciELO) e na Biblioteca Nacional de Medicina dos EUA / Institutos Nacionais de Saúde (PubMed). Utilizou-se os seguintes descritores em português e inglês "Eletroacupuntura", "Incontinência Urinária por esfresse," "Eletroacupuncture", "Stress Urinary Incontinence" constantes nos Descritores 


\section{artigo}

Carvalho, P.C.; Barreiros, R.N.; Dutra, L.B.; Mouta, R.J.O.; Louro, T.Q.;

Evidências do Tratamento de Eletroacupuntura em Mulheres com Incontinência Urinária de Esforço: Uma revisão integrativa

em Ciências da Saúde (DeCs) e o operador booleano AND.

Adotados os seguintes critérios de inclusão dos artigos: textos completos e com resumos disponíveis em português e inglês, estudos realizados em humanos classificados como ensaios clínicos, ensaios clínicos controlados e randomizados, artigos clássicos, relatos de caso e estudos observacionais. Excluídos da análise as publicações tipo: Editoriais, ponto de vista ou reflexão, artigos de revisão da literatura, dissertações e teses. Os artigos que apareceram em mais de uma base de dados, utilizou-se apenas um para ser submetido à análise. As etapas desta revisão estão sintetizados e apresentados sob a forma de um fluxograma na figura 1 , utilizando o modelo PRISMA`.

\section{RESULTADOS}

Foram localizados 20 artigos nas bases de dados. Após a leitura dos títulos e resumos e a aplicação dos critérios de inclusão e exclusão resultou em 5 artigos. Em seguida, após a leitura
Figura 1. Fluxograma explicativo da seleção dos artigos. Rio de Janeiro/RJ,2020.

Registros identificados por meio de

pesquisa no bando de dados MEDLINE,

LILACS, SCIELO, PUBMED $(n=20)$
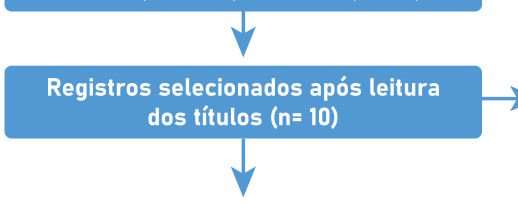

Registros selecionados após leitura do resumo $(n=08)$
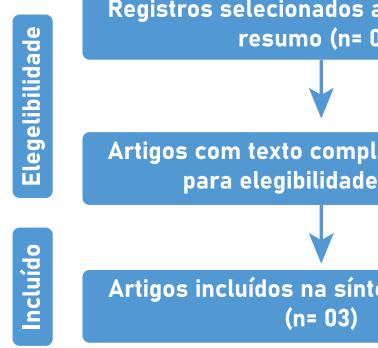

Artigos com texto completos avaliados para elegibilidade $(n=05)$

Artigos incluídos na síntese qualitativa ( $n=03$ ) na íntegra, obteve-se um total de 3 artigos para realizar o embasamento bibliográfico.

Os artigos foram publicados entre os anos de 2016 a 2019, todos feitos na China e escritos em inglês. Um resultado preliminar observado é certamente a escassez de publicações que investigam a terapia de eletroacupuntura no tratamento da IUE.

Especificações da qualidade metodológica, da amostra, da intervenção e resumo dos resultados dos três artigos ${ }^{9,10,11}$ estão resumidos na tabela 1 . Todas as participantes dos estudos foram atendidas em ambulatório e separadas em grupo da eletroacupuntura (EA) ou da falsa eletroacupuntura, conhecida como sham eletroacupuntura $(\mathrm{SA})^{9,10}$. A SA é a maneira metodológica que muitos ensaios clínicos usam para estabelecer o grupo controle. Escolhem pontos fora dos meridianos da acupuntura e para garantir o mascaramento utilizam agulhas placebo que são semelhantes com as agulhas convencionais, porém confeccionadas com um sistema que as impede de penetrar na pele ${ }^{12}$. Nos grupos SA dos dois ensaios clínicos encontrados foram utilizadas as agulhas placebo, associadas aos acupontos falsos e os cabos que conduziriam os estímulos elétricos estavam rompidos.

Os pontos selecionados no meridiano da Bexiga, o B33 e B35, foram selecionados por suas indicações baseadas na teoria da MTC e pela localização da inervação do sistema uri-

\section{Tabela 1. Características dos estudos incluídos na revisão integrativa, Rio de Janeiro/RJ,2020}

\begin{tabular}{|c|c|c|c|c|}
\hline Estudo & Ano & Tipo e Estudo & Amostra & Interveção \\
\hline $\begin{array}{l}\text { Xu H } \\
\text { et al. }{ }^{9}\end{array}$ & 2016 & $\begin{array}{l}\text { Piloto, ensaio clínico } \\
\text { randomizado, placebo, } \\
\text { controlado, cego para } \\
\text { participante }\end{array}$ & $\begin{array}{l}80 \text { mulheres } \\
\text { idade } 40-75 \\
\text { anos }\end{array}$ & $\begin{array}{l}18 \text { sessões, } 30 \text { min cada, } \\
6 \text { semanas de tratament, } \\
3 x \text { por semana. Follow up } \\
\text { de } 24 \text { semanas. Grupo EA }\end{array}$ \\
\hline $\begin{array}{l}\text { Liu Z } \\
\text { et al. } 10\end{array}$ & 2017 & $\begin{array}{c}\text { Ensaio Clínico, multicên- } \\
\text { trico, randomizado, cego } \\
\text { para participante }\end{array}$ & $\begin{array}{l}504 \text { mulheres } \\
\text { idade 40-75 } \\
\text { anos }\end{array}$ & $\begin{array}{c}\text { usou pontos B33 e B34. } \\
\text { Grupo SA, uso de agulhas } \\
\text { placebo distante } 20 \mathrm{~mm} \\
\text { dos pontos B33 e B34. } \\
\text { Parâmetros de EA 50Hz, } \\
\text { 1-5mA. Mesmo proto- } \\
\text { colo de intervenção de } \\
\text { Huafang Xu et al. }\end{array}$ \\
\hline $\begin{array}{l}\text { Jiao R } \\
\text { et al. }{ }^{11}\end{array}$ & 2019 & $\begin{array}{c}\text { Estudo de Coorte Obser- } \\
\text { vacional }\end{array}$ & $\begin{array}{l}349 \text { mulhes } \\
\text { na pós meno- } \\
\text { pausa }\end{array}$ & $\begin{array}{c}\text { Análise pós-hoc e análise } \\
\text { de regressão logística } \\
\text { com os dados do estudo } \\
\text { de Zhishun Liu et al. }\end{array}$ \\
\hline
\end{tabular}

\section{Resultado}

Redução do volume de perda de urina, grupo EA no pad-test $1 \mathrm{~h}(\mathrm{p}=0.01)$. Redução dos episódios de perda de urina do DM no gruopo EA $(p<0,05)$ diferença significativa entre grupo na $24^{\circ}$ semana do follow up, ICIQ-SF diferença significativa entre EA e SA $(p<0,05)$. Diferença estátistica entre os grupos $(p<0,01)$ no pas test $1 \mathrm{~h}$. Grupo AE perda menor de volume de urina. No DM diferença estátistica entre os grupos $(p<0,01)$ no follow up ICIQ-SF diferença estátistica entre os grupos $(p<0,01)$ na $6^{\circ}$ semana de tratamento e no follow up Mulheres no grupo EA tiveram menos perda de volume urina no pad test $1 \mathrm{~h}$ entre $2^{\circ}$ e $6^{\circ}$ semana de tratamento em comparação ao grupo SA $(p<0,01)$. Responderam melhor ao tratamento de EA e SA as mulheres que usavam menos absorventes por semana 
nário na medicina ocidental ${ }^{9,10}$. Em ambos estudos, os grupos de EA e SA realizaram manipulaçôes nas agulhas após as inserções verdadeiras ou falsas com intenção de obter a sensação do De Qi, definido como um conjunto de sensações que podem ser descritos pelo paciente (como dor, dormência, peso ou outras sensações locais) no momento da manipulação da agulha ${ }^{10}$.

\section{DISCUSSÃO}

Após a leitura dos artigos foi realizada uma análise que dividiu em categorias para uma melhor avaliação dos resultados encontrados. Nessa divisão foi possível observar os seguintes assuntos: Avaliação dos sintomas da perda de urina antes e após o tratamento com eletroacupuntura utilizando o pad test 1h, análise do Diário Miccional (DM) de $72 \mathrm{~h}$, avaliação da qualidade de vida com o questionário ICIQ-SF, os acupontos selecionados e os parâmetros da eletroacupuntura.

Avaliação do pad test 1h: O pad test é um teste de fácil aplicabilidade, objetivo e válido para a quantificação do volume da perda da urina. Consiste em usar um absorvente (pad) por um tempo de 1 hora previamente pesado e o colocar próximo do meato uretral externo $^{13}$. Após esse tempo, ele será repesado e verificado se houve aumento do seu peso pela perda da urina. Nos dois ensaios clíni$\cos ^{9,10}$ foi constatado que os grupos de EA em comparação aos grupos de SEA obtiveram uma maior redução da perda de urina.

$\mathrm{O}$ estudo de Jiao et al..$^{11}$ também encontrou resultado semelhante. Porém, as participantes que tinham maiores quantidades de perdas não obtiveram melhoras com o tratamento da EA. Assim, pode-se supor que a EA seria uma opção complementar aos outros tratamentos convencionais, como o treino dos músculos do assoalho pélvico (TMAP).

Análise do Diário Miccional (DM) de 72h: O Diário Miccional é um instrumento usado para avaliar os hábitos urinários de pessoas com sintomas de incontinência urinária $(\mathrm{IU})^{14}$. Nos resultados encontrados, os grupos de EA, apresentaram maiores reduções dos episódios de IU, principalmente no fim do follow-up. Uma possível explicação feita pelos autores seria o efeito de longa duração e acumulativa da $\mathrm{EA}^{9,10}$. Paik et al. ${ }^{15}$ observaram que em alguns casos a acupuntura melhorou os sintomas $\mathrm{da}$ incontinência urinária. Contudo, na análise não discriminaram o tipo de IU (de esforço ou mista, por exemplo) e concluíram que ainda não haveria evidência suficiente para explicar a ação da acupuntura nessa melhora. O estudo de Bergström et al. ${ }^{16}$ investigou o uso da acupuntura em idosas com IU de urgência e mista. A explicação dos autores para a melhora dos sintomas foi provavelmente aos mecanismos fisiológicos da aplicação da acupuntura que aumentou a quantidade de $\beta$-endofirnas no SNC e teria

\section{Apesar de}

reconhecer a

importância da

integridade dos

músculos do

assoalho pélvico

(MAP) por manter

os mecanismos da

continência durante

os esforços, deve-se

destacar também

a necessidade

de um sistema

neuromuscular

funcional e intacto. inibido o centro pontino de micção provocando o aumento da reserva da bexiga durante a fase de enchimento.

Apesar de reconhecer a importância da integridade dos músculos do assoalho pélvico (MAP) por manter os mecanismos da continência durante os esforços, deve-se destacar também a necessidade de um sistema neuromuscular funcional e intacto. Yang et al. ${ }^{17}$ mostraram com o ultrassom, como a atividade reflexa dos MAP é importante para manter a continência pelo aumento da pressão intrauretral. Entretanto, os autores explicaram que a literatura ainda é escassa em poder explicar como seria o funcionamento desse reflexo.

Qualidade de vida ICIQ-SF: O ICIQ-SF é um questionário autoaplicável, específico para avaliar o impacto da IU na qualidade de vida dos pacientes ${ }^{18}$. Nos dois ensaios clínicos o grupo de EA apresentou redução no score do ICIQ-SF mostrando menor impacto da IU na qualidade de vida. Esse resultado foi significativo durante o tratamento e mantido no follow-up. O estudo de Jiao et al. ${ }^{11}$, no entanto, não encontrou diferenças utilizando o ICIQ-SF. Pelo exposto, há evidências de melhora da qualidade de vida com o tratamento da EA na IUE e essa resposta é fundamental pelos efeitos negativos da IU no âmbito psicológico, físico, social, sexual e ocupacional ${ }^{19}$.

Acupontos e Parâmetros de Eletroacupuntura: Os acupontos selecionados foram o B33 e B35. Os autores ${ }^{9,10}$ explicaram que a escolha dos dois acupontos foi devido a IUE estar relacionada com a disfunção da bexiga pela MTC e por estarem localizados na região lombossacra. A localização fica proximalmente sobre o ramo posterior de S3, correspondendo a localização do nervo pudendo que tem origem nos segmentos S2-S4 da medula e controla o esfincter externo da uretra e os músculos do assoalho pélvico ${ }^{20}$. Outros estudos avaliaram mais acupontos na região sacral como o B31, $\mathrm{B} 32 \mathrm{e} \mathrm{B} 33^{16}$ ou pontos na região pélvica ventral como os pontos $\mathrm{VC} 3, \mathrm{VC} 4 \mathrm{eVC} 6^{15}$. Em outro estudo mais recente, Liu et al. ${ }^{21}$ selecionaram os mesmos pontos B33 e B35 para tratamento de pacientes com IU mista. Portanto, parece uma tendência a escolha de pontos do meridiano da Bexiga próximos a emersão do nervo pudendo. No entanto, ainda não se sabe se outros pontos da Bexiga ou mesmo do meridiano do 


\section{artigo}

Carvalho, P.C.; Barreiros, R.N.; Dutra, L.B.; Mouta, R.J.O.; Louro, T.Q.;

Evidências do Tratamento de Eletroacupuntura em Mulheres com Incontinência Urinária de Esforço: Uma revisão integrativa

Rim, acoplado da Bexiga segundo a MTC e com função de governar o elemento água ${ }^{4}$ teria efeito no tratamento na IUE. Em relação aos parâmetros da EA, os estudos ${ }^{9,10}$, utilizaram os parâmetros de $50 \mathrm{~Hz}$ de frequência, com onda contínua, intensidade da corrente de $1-5 \mathrm{~mA}$ e 30 minutos de tratamento. Subentendeu-se que os pulsos usados provavelmente foram do tipo "não polarizado" por não descreverem a localização dos pólos. A frequência de $50 \mathrm{~Hz}$ para a acupuntura energética é considerada alta, pois se situa na faixa de 50 à $2500 \mathrm{~Hz}^{5}$. Os estudos não expuseram a razão da frequência escolhida e assim não se pode dizer qual foi o critério para essa escolha. Talvez, a opção pela frequência de $50 \mathrm{~Hz}$ foi com a intenção de estimular eletricamente provocando uma despolarização da terminação nervosa, semelhante como se usa no tratamento de Fisioterapia Pélvica, para reforçar o trabalho muscular do assoalho pélvico ${ }^{22}$. Assim, ainda não seria possível estabelecer nesse momento quais seriam os melhores parâmetros mais eficazes para a terapia de eletroacupuntura na IUE.

\section{CONCLUSÃO}

Apesar dos poucos trabalhos publicados, parece que existe uma tendência na melhora dos sintomas de IUE utilizando a eletroacupuntura. Os resultados até esse momento mostraram relevante melhora nos sintomas que merecem maiores investigações.

\section{REFERÊNCIAS}

1. Minassian VA, Drutz HP, Al-Badr A. Urinary incontinence as a worldwide problem. Int J Gynaecol Obstet 2003 Sep; 82(3):327-338.

2. Haylen BT, de Ridder D, Freeman RM, Swift SE, Berghmans B, Lee J, et al. An International Urogynecological Association (IUGA)/ International Continence Society (ICS) joint report on the terminology for female pelvic floor dysfunction Review Article. Neurourol and Urodyn 2010; 29(1): 4-20.

3. Sun Z, Yu J, Zhang Q. Acupuncture for urinary incontinence after stroke: a protocol for systematic review. BMJ Open 2016 Feb 23; 6(2):1-5.

4. Maciocia, G. Os fundamentos da Medicina Chinesa: Um texto abrangente para acupunturistas e fitoterapeutas. 3. ed. São Paulo: Roca, 2017.

5. Athayde FB. Eletroacupuntura: Fundamentos para a prática clínica. 2. ed. rev. e ampl. São Paulo: Andreoli, 2019.

6. Mendes KDS, Silveira RCCP, Galvão CM. Revisão Integrativa: Método de Pesquisa para a incorporação de evidências na saúde e na enfermagem. Texto Contexto Enferm 2008 Florianópolis; Out-Dez; 17(4): $758-764$.

7. Botelho LLR, Cunha CCA, Macedo M. O método da revisão integrativa nos estudos organizacionais. Gestão e Sociedade 2011 Belo Horizonte; 11(5): 121-136.

8. Santos CMDC, Pimenta CADM, Nobre MRC. A estratégia PICO para a construção da pergunta de pesquisa e busca de evidências. Rev Lat Am Enfermagem 2007, 15(3), 508-511.

9. Xu H, Liu B, Du R, Liu X, Yu J, Liu Z. A pilot randomized placebo controlled trial of eletroacupuncture for women with pure stress urinary incontinence. PLoS One 2016 mar 9; 11(3):1-8.

10. Liu Z, Liu Y, Xu H, He L, Chen Y, Fu L, et al. Effect of eletroacupuncture on urinary leakage among women with stress urinary incontinence. A Randomized Clinical Trial. JAMA 2017 Jun 27; 317(24): 24932501.

11. Jiao R, Liu Y, Liu B, Liu Z. Risk factors related to acupuncture response in postmenopausal women with stress urinary incontinence. Medicine (Baltimore) 2019 Apr; 98(16): 1-5.

12. Lopes-Júnior LC, Cruz LAP, Leopoldo VC, Campos FR, Almeida AM, Silveira RCCP. Efetividade da Acupuntura Tradicional Chinesa versus Sham Acupuntura: Revisão Sistemática. Rev Lat Am Enfermagem
2016 Aug; 24:1-12.

13. Micussi MTABC, Soares EMM, Lemos TMAM, Brito TNS, Silva JB. Correlação entre as queixas de incontinência urinária de esforço e o pad test de uma hora em mulheres pós menopausa. Rev Bras Ginecol Obstet 2011; 33(2): 70-74.

14. Oliveira NFF, Marques AA, Frederice CP, Fogaça NMM. Avaliação fisioterapêutica na incontinência urinária. In: Pinto e Silva MP, Marques AA, Amaral MTP. Tratado de fisioterapia em saúde da mulher. 2 ed. Rio de Janeiro: Roca; 2019. 289-304.

15. Paik SH, Han SR, Kwon OJ, Ahn YM, Lee BC, Ahn SY. Acupuncture for the treatment of urinary incontinence: $A$ review of randomized controlled trials.Exp Ther Med 2013; 6(3): 773-780.

16. Bergström K, Carlsson CPO, Lindholm C, Widengren R. Improvement of urge - and mixed - type incontinence after acupuncture treatment among elderly women - a pilot study. J Auton Nerv Syst 2000; 79: 173-180.

17. Yang JM, Yang SH, Huang WC, Tzeng CR. Factors affecting reflex pelvic floor muscle contraction patterns in women with pelvic floor disorders. Ultrasound Obstet Gynecol 2013; 42: 224-229.

18. Tamanini JTN, Dambros M, D'ancona CAL, Palma PCR, Rodrigues Netto Jr N. Validação para o português do "International Consultation on Incontinence Questionnaire Short Form" (ICIQ-SF). Rev Saúde Pública [Internet]. 2004 Jun [acesso em 25 Jun 2019]; 38(3): 438444.

19. Borba AMC, Lelis MAS, Bretas ACP. Significado de ter incontinência urinária e ser incontinente na visão das mulheres. Texto Contexto - Enferm 2008; 17(3): 527-535.

20. Yoshimura N, Miyazato M. Neurophysiology and therapeutic receptor targets for stress urinary incontinence. Int J Urol 2012 Jun; 19(6): 524-537.

21. Liu B, Liu Y, Qin Z, Zhou K, Xu H, He L, et al. Eletroacupuncture versus pelvic floor muscle training plus solifenacin for women with mixed urinary incontinence: $A$ randomized noninferiority trial. Mayo Clin Proc 2019; 94(1): 54-65.

22. Oliveira NFF, Marques AA, Frederice CP, Fogaça NMM, Bardin MG. Recursos fisioterapêuticos e aplicabilidade ao tratamento da incontinência urinária. In: Pinto e Silva MP, Marques AA, Amaral MTP. Tratado de fisioterapia em saúde da mulher. 2 ed. Rio de janeiro: Roca; 2019. 305-319. 